\title{
Programas de inserción laboral para personas con discapacidad auditiva: una experiencia concreta en el Principado de Asturias*
}

\section{Programs of job integration for persons with hearing disability: a concrete experience in the Principality of Asturias}

\section{Mํㅡㄹ DE LOS ÁNGELES FLÓREZ GARCÍA ${ }^{1}$}

\begin{abstract}
RESUMEN
En este articulo se expone un programa concreto de empleo para personas con Deficiencia Auditiva en el Principado de Asturias (España). Los procedimientos y datos que se exponen, a continuación, se han desarrollado entre los años 1998 y 2002. Nuestro trabajo, fundamentalmente, se ha centrado en jóvenes con deficiencia auditiva, sus familias y empresas en general, aunque también hemos trabajado en otros ámbitos.

Los resultados demuestran, a nuestro modo de ver: 1) que las personas con deficiencia auditiva tienen capacidad para competir con éxito en el mercado de trabajo ordinario, en virtud de sus propios méritos con un poco de asesoramiento y apoyo y 2) la necesidad de estabilidad de estos proyectos dada la progresión de los resultados obtenidos.
\end{abstract}

\section{PALABRAS CLAVE}

Inserción laboral, Discapacidad auditiva, Mercado de trabajo, Ámbitos de empelo.

* Elaborado por Ma de los Ángeles Flórez García en base a la "Guía de Buenas Prácticas para los Servicios de Empleo” de la Red de Inserción Laboral de FIAPAS. Madrid, 2004, FIAPAS. Y de la legislación básica vigente en el momento de desarrollo del Proyecto.

${ }^{1}$ Piscóloga colegiada 0-1105. 


\section{ABSTRACT}

This issue features a specific employment program for persons with hearing impairment taking place in Asturias (Spain). The procedures and dates that follow occurred between 1998 and 2002. Our job focused primarily on young hearing impaired persons, their families and non-specifically targeted companies, but we also have worked in other areas.

We believe that the results achieved show: 1) that hearing impaired persons can compete successfully in the work market on their own merits and needing only little advice and support and 2) and need stability for these projects based on the excellent results achieved.

\section{KEY WORDS}

Job integration, Hearing disability, Work market, Working areas. 


\section{INTRODUCCIÓN:}

La inserción laboral de personas con discapacidad, y en concreto, de personas con discapacidad auditiva es la vía de normalización e integración social que corresponde a la etapa adulta de cualquier persona y por ende a las personas con discapacidad. Máxime si tenemos en cuenta los esfuerzos extras de las personas con discapacidad, de sus familias y de los poderes públicos a lo largo de todas sus etapas vitales previas.

Para garantizar estos derechos, desde el punto de vista legal, la propia Constitución española, como ley de referencia, en sus artículos 9.2 y 49 propone determinados derechos constitucionales específicamente para las personas con alguna discapacidad.

Más tarde y como desarrollo de la Constitución española es promulgada la Ley 13/1982, de 7 de Abril, de Integración Social de los Minusválidos (LISMI). La citada ley dedica su Título VII a la Integración Laboral y propone las vías adecuadas para conseguir la citada integración.

A raíz de todas estas normativas básicas y en el contexto de los años 90, surgen diversos programas para la integración laboral de personas con discapacidad, llevados a cabo a través de diversos organismos y asociaciones y subvencionados, en su mayor parte, mediante fondos europeos.

Dentro de este contexto se pone en marcha el programa que ocupa este artículo en la Asociación de Padres y Amigos de Deficientes Auditivos (APADA-ASTURIAS). Dicha Asociación tiene como fin primordial la atención a las personas con deficiencia auditiva en todas las etapas de su vida, incluida la integración en el medio educativo y laboral de los jóvenes con deficiencia auditiva. Así, surge el Servicio de Información y Orientación para el Empleo de las personas Sordas (S.I.O.S.). En su inicio, dicho servicio, se enmarcó en los Proyectos de Inserción Laboral, convocados (Orden de 23 de noviembre de 1998, BOE 26 de enero de 1999) y subvencionados por el MEC (Orden 28 de abril de 1999, BOE 27 de mayo de 1999). De esta forma, el SIOS inicia su andadura en 1998, para responder a las necesidades de formación y de inserción laboral de los jóvenes con deficiencia auditiva del Principado de Asturias.

El punto de partida se centra en que a pesar de que el empleo para las personas con discapacidad sigue en continuo crecimiento. El "Plan de Acción para el Empleo del Reino de España 1998" refleja que se ha pasado de unos cientos de contratos indefinidos en 1983 a 5.725 contratos indefinidos para personas con minusvalía en 1997. Sin embargo, la tasa de desempleo todavía es mayor entre las personas con discapacidad que entre la población general. Concretamente 40.000 personas con discapacidad estaban inscritas en las oficinas del INEM como demandantes de empleo en 1997.

Por otra parte, los empleos a los que suelen acceder las personas con discapacidad son de escasa cualificación y retribución, debido en parte a sus bajos niveles de formación pero también a los prejuicios de algunos empresarios que no les consideran capacitados para acceder al mundo de la empresa o para promocionarse u ocupar empleos de más responsabilidad.

Los objetivos fundamentales de trabajo en el SIOS de APADA-ASTURIAS son los siguientes:

- La orientación de los jóvenes sordos que suelen presentar carencias 
formativas y de cualificación profesional o incluso personal, que en muchas ocasiones dificulta su ajuste a la realidad del mercado laboral y su acceso al empleo.

- La necesidad de inserción laboral en empresa ordinaria, es decir, en un medio de trabajo normalizado, como vía para la inserción social y la independencia económica y personal de las personas con deficiencia auditiva.

- La eliminación de barreras a la contratación de personas con deficiencia auditiva, provocadas, en su mayor parte por el gran desconocimiento que existe en la sociedad y en el empresario como parte de ella, de las aptitudes y capacidades del joven con deficiencia auditiva. Con el fin de lograr este objetivo, el SIOS, fundamentalmente ha dirigido sus esfuerzos a:

1. La mentalización y sensibilización de empresarios dándoles a conocer las aptitudes y capacidades de las personas sordas y las ventajas de su contratación.

2. La detección y análisis de las dificultades de comunicación con otros trabajadores y directivos oyentes, con el objetivo de anticiparse a ellas y evitarlas o solventarlas una vez que se han producido.

3. En general, los educadores, compañeros de trabajo, los empresarios, sindicatos y todos los agentes que puedan estar implicados en el proceso de inserción laboral de los jóvenes con deficiencia auditiva, deben conocer una serie de aspectos básicos fundamentales que APADA pretende difundir con el desarrollo de los Proyectos de Empleo para sensibilizar a la sociedad en su conjunto.

De forma más detallada nuestro trabajo concreto tenía un recorrido y tuvo unos resultados que pasamos a exponer a continuación.

\section{DESCRIPCIÓN DEL ITINERARIO DE INSERCIÓN LABORAL}

La llegada de las personas con discapacidad auditiva a los Servicios de Empleo suele ser, en muchos casos, el primer paso de su Itinerario de Inserción laboral. De ahí, la importancia que tiene en este primer encuentro la acogida que se preste a los usuarios demandantes de empleo.

Esta acogida debe tener en cuenta:

- Que las dificultades que limitan la comunicación de las personas sordas inciden directamente en las relaciones interpersonales, afectando a las actividades que realizan en el entorno laboral.

- Que la mayoría de las personas sordas que acuden al Servicio de Empleo suelen estar desorientadas, desmotivadas, poco familiarizadas con la realidad del mercado laboral, disponen de poca información, etc.

- Que son muchas las circunstancias que conforman la situación de partida de cada usuario, que varía según su situación familiar, económica, emocional, formación, experiencia, etc.

- Que el objetivo de la persona sorda que acude al Servicio de Empleo es 

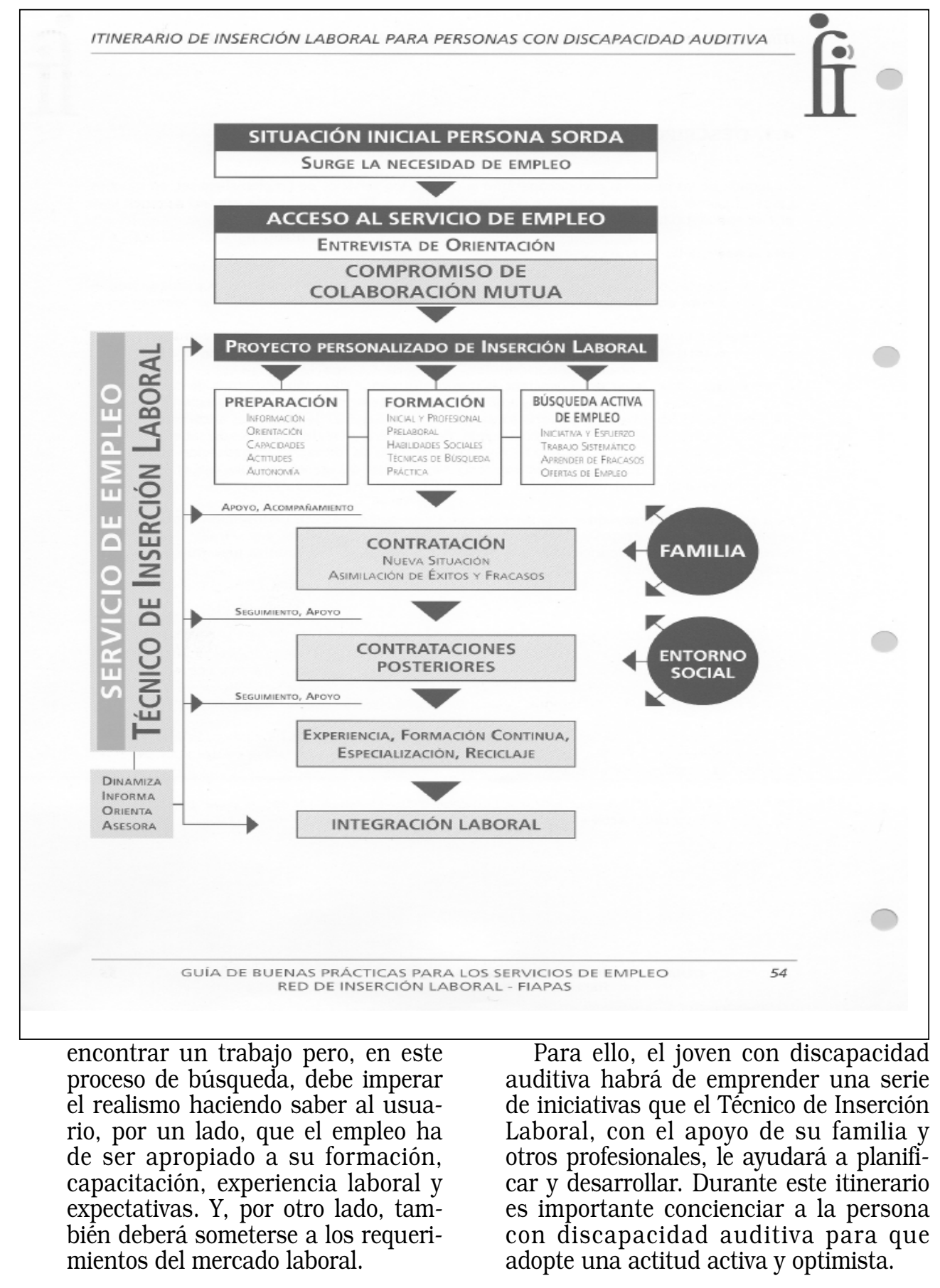


\section{Preparación de los jóvenes sordos para la inserción laboral}

La primera entrevista que el joven sordo realiza con el Técnico de Inserción laboral es crucial, ya que en ella es necesario ganarse la confianza del usuario para colaborar mutuamente, analizar la situación de partida, diseñar un plan personal de búsqueda de empleo y ponerlo en práctica. El joven debe aceptar el reto y asumir el compromiso.

El Técnico de Inserción laboral escucha, informa, orienta, motiva, planifica y ayuda a llevar a cabo el plan personal de búsqueda de empleo.

En primer lugar, el usuario facilitará al Técnico de Inserción laboral información sobre su formación, conocimientos, cualificación, capacidades, aptitudes, etc. Asimismo, es importante que en este primer contacto se obtenga información acerca de la situación personal del usuario para poder situar sus necesidades inmediatas, disponibilidad, actitudes, calibrar sus expectativas, etc. Por último, es necesario que el Técnico de Inserción laboral fomente la reflexión del usuario sobre sus preferencias, habilidades, fortalezas y debilidades, objetivos profesionales, etc. Toda esta información es necesaria a la hora de diseñar y establecer un plan personal de inserción laboral.

El Técnico debe procurar que el usuario no se angustie ante las dificultades que puedan surgir, dotándole de todos los instrumentos necesarios para la búsqueda de empleo, incluyendo el aprendizaje de la correcta elaboración del currículum vitae, la carta de presentación y la organización del plan de búsqueda de empleo personalizado.

Asimismo, proporcionará al usuario la información necesaria sobre los recursos existentes en materia de empleo: fuentes de empleo, técnicas de búsqueda de empleo, situación del mercado de trabajo, ofertas apropiadas a su perfil, etc. Informará sobre el entorno laboral para su mejor conocimiento, tratando de fomentar hábitos laborales necesarios para un correcto desarrollo profesional.

Además, cuando el usuario se enfrente a un el proceso de selección. El técnico analizará cada una de las acciones que se realicen, desde el primer contacto hasta la incorporación al puesto de trabajo. Para preparar la entrevista de selección, es conveniente la realización de entrevistas simuladas a fin de que el usuario adquiera soltura y práctica. Así mismo, es necesario que se establezcan las pautas a seguir durante las primeras semanas en el puesto de trabajo para conseguir una mejor adaptación y la integración en la empresa.

El Técnico debe colocar al usuario en disposición de utilizar todas las herramientas a su alcance, aprovechando su preparación, motivación, dedicación y perseverancia para la consecución del objetivo final, la obtención de un puesto de trabajo.

En definitiva, el Técnico de Inserción laboral debe inducir y colaborar con el usuario en la realización de una búsqueda activa de empleo.

Como Técnico de Inserción laboral de APADA-Asturias, gestioné una "Bolsa de empleo", es decir de usuarios con deficiencia auditiva, de 114 personas. En su mayoria jóvenes, dado que se trata de una Asociación de Padres de Personas con Deficiencia Auditiva y que en su origen, como ya hemos reseñado anteriormente, fue un servicio subvencionado a través del MEC. 
Con este fin, desde el SIOS se trabaja en sesiones individualizadas cuando los usuarios acceden a nuestro servicio haciendo un análisis exhaustivo de sus características profesionales e incluso personales y tratando de aportarles las herramientas necesarias para que ellos mismos participen de forma activa en la búsqueda de empleo.

En este mismo sentido, se imparten cursos colectivos de Habilidades Sociales y Preelabórales, para todo usuario que se ha interesado en participar en dichos cursos.

Sin embargo, mi función como Técnico de Inserción Laboral no termina una vez conseguida la inserción laboral del usuario, sino continua con el seguimiento del puesto de trabajo. Así, se prestará apoyo al trabajador insertado y al empresario, se detectarán posibles deficiencias del entorno laboral de la persona con discapacidad auditiva, etc. De esta manera, se podrán aplicar medidas correctoras con el fin de lograr estabilizar cada inserción laboral.

Será necesario diseñar una estrategia de seguimiento adecuada a cada puesto de trabajo. Para esto, es imprescindible conocer las características de la empresa, de sus responsables, de los compañeros de trabajo, así como las del usuario, y emplear los medios más apropiados para cada caso, como pueden ser: visita a la empresa, llamada telefónica, contacto por correo electrónico, etc. Siempre que la empresa lo permita, la visita en el puesto de trabajo nos ofrecerá una visión más real de la situación y una mejor información sobre otros aspectos que no es posible detectar por otros medios.

Otra información que es imprescindible conocer para el correcto apoyo y seguimiento es el grado de satisfacción de la persona con discapacidad auditiva, de los compañeros, del empresario y de la familia.

Por último, a través del seguimiento del trabajador insertado, se debe analizar también su situación en cuanto a experiencia adquirida, rendimiento, sueldo, etc., con el fin de ver las posibilidades de promoción dentro de la empresa 0 de mejora de empleo en otra empresa. Por tanto, otro frente de trabajo, es la empresa en general, que se gestionaba desde el SIOS, como pasamos a exponer en el siguiente apartado.

\section{ÁMBITOS DE EMPLEO}

Las empresas y por tantos los posibles empleos y forma de acceso a ellos son diferentes en función, fundamentalmente de que pertenezcan al ámbito público 0 privado y por tanto hay que diferenciar claramente este concepto antes de plantearse uno u otro campo de empleo.

\section{Acceso al empleo privado}

El empleo privado u ordinario es el empleo generado por empresas normalizadas en las que el porcentaje mayoritario de trabajadores está compuesto por personas sin discapacidad y está sujeto a la libre oferta y demanda del mercado de trabajo. A este campo de trabajo, es decir a este tipo de empresas es a las que fundamentalmente hemos dedicado nuestro trabajo. Teniendo en cuenta que el mercado de trabajo actual se caracteriza por: fuerte competitividad, alta tasa de temporalidad, demanda de formación cada vez más especializada, polivalencia en muchos puestos de trabajo, etc.

Por esta razón, previo a iniciar una prospección empresarial, es importante definir los objetivos que se persiguen y 
hacer una buena planificación y programación de las acciones a llevar a cabo.

Acciones generales a desarrollar con las empresas:

- Prospección empresarial de la zona de intervención. Consiste en la recogida de información sobre las características, comportamiento, necesidades, intereses y perspectivas del tejido empresarial de una determinada zona.

- Diseño del plan de comunicación a las empresas (visita a las instalaciones, análisis y detección de posibles puestos de trabajo, información sobre las modalidades contractuales y establecimiento de posibles vías de colaboración).

- Fomento de la implicación empresarial y sindical en la integración laboral de las personas con discapacidad auditiva.

- Captación y análisis de las ofertas de trabajo y preselección de los posibles candidatos.

- Seguimiento de la inserción laboral de las personas con discapacidad.

Desde nuestro SIOS se ha mantenido contacto directo con 210 empresas del Principado de Asturias que en algún momento han aceptado recibir información detallada sobre posibles contrataciones a personas con deficiencias auditivas. Dicha información inicial consta, básicamente, de las posibilidades de empleo de nuestros usuarios, de sus capacidades productivas y de las ventajas fiscales, de descuentos en seguridad social o subvenciones previstas por la contratación a personas con discapacidad. En algunas ocasiones y tras analizar las necesidades de la empresa se han producido, de forma inmediata o demorada en el tiempo, ofertas concretas. A las que hemos presentado alguno 0 varios de nuestros usuarios en función de las demandas de la empresa y de la disponibilidad e interés de nuestros usuarios. Concretamente nuestro sistema de trabajo es el que pasamos a exponer de forma más detallada.

El acercamiento a la empresa, puede realizarse por diferentes vías:

- Por iniciativa propia del Servicio de Empleo, éste oferta sus servicios a una empresa en concreto para poner a su disposición los perfiles profesionales específicos en la actividad a la cual se dedica la empresa.

- A partir de una oferta concreta, de la prensa, Oficina de Empleo, Empresas de Trabajo Temporal (ETT), etc., el Servicio se pone en contacto con la empresa para cubrir la oferta.

- A partir de una acción emprendida por un demandante de empleo por iniciativa propia.

- Por la solicitud de información de una empresa como consecuencia de las acciones de divulgación del Servicio de Empleo.

En función de los cuatro supuestos anteriores, las vías de acceso a la empresa variarán, pero el objetivo final será igual para los cuatro. Es decir, encontrar un trabajo que pueda desarrollar el demandante de empleo.

El procedimiento habitual consiste en:

- Mailing dirigido a un grupo de empresas seleccionadas en función del sector al que pertenecen, activi- 
dad que desarrollan y localización territorial. Se trata del envío de una carta introductoria del Servicio de Empleo, en la cual se ofrecen las prestaciones del mismo y se informa que posteriormente se efectuará una llamada telefónica por parte de un Técnico de Inserción laboral con el objetivo de ampliar la información.

- Dejando un tiempo de margen, se procede a la llamada telefónica con el objetivo de conseguir una entrevista presencial en la empresa para, por un lado, conocer sus principales características y analizar posibles puestos de trabajo, y por otro, ofrecer las prestaciones del servicio, detectando y clarificando posibles ideas erróneas del colectivo al cual representamos.

- Una vez en la empresa, se realiza una detección de los puestos de trabajo susceptibles de ser ocupados por personas con discapacidad auditiva. En el análisis del puesto de trabajo se recoge toda la información necesaria y se procede a establecer vías de colaboración para el futuro o bien se gestionan las ofertas de trabajo que puedan surgir.

En el caso de que se produzca una oferta de empleo clara por parte de la empresa, la labor de intermediación laboral del Servicio de Empleo, se centra en las siguientes acciones:

- Análisis exhaustivo del puesto de trabajo a cubrir en la empresa.

- Información sobre los candidatos de la bolsa de empleo.

- Preselección de candidatos.

- Información sobre la legislación relativa a la contratación de trabaja- dores con discapacidad. Asesoramiento sobre adaptaciones del puesto de trabajo, ayudas económicas y técnicas de la Administración en caso de que sea necesario.

- Módulo informativo para los trabajadores de la empresa.

- Apoyo en el lugar de trabajo en caso necesario.

- Seguimiento de la inserción.

Es importante tener siempre en cuenta que todas las acciones de intermediación que se produzcan, tanto de información del candidato al empresario, como a la inversa, han de contar con el consentimiento del interesado en cualquiera de los casos.

Tras la contratación por parte de la empresa, se inicia un proceso de seguimiento en el puesto de trabajo que incluye tanto a la empresa como al trabajador contratado. Este seguimiento puede ser muy variable en el tiempo, pero siempre deberá contemplar la evaluación continua de:

- Condiciones del trabajo.

- Rendimiento en el puesto de trabajo.

- Nivel de satisfacción del empresario y del trabajador.

- Relaciones sociales y comunicación que tienen lugar en el desempeño del trabajo.

- Posibilidades de promoción y mejora laboral.

- Necesidades de formación específica generadas para el desempeño del puesto. 
Para que el seguimiento del puesto de trabajo sea efectivo, se pueden realizar entrevistas de seguimiento al trabajador fuera de su horario de trabajo, visitas a las empresas 0 todas aquellas acciones que se estimen oportunas (cuestionario al trabajador 0 al empresario, etc.).

Es importante destacar que, a lo largo del seguimiento, se debe poder ofrecer a la empresa el módulo de informaciónformación sobre el colectivo de personas con discapacidad auditiva, clarificando posibles dudas en cuestión de comunicación, pautas de relación con una persona sorda, ayudas técnicas, adaptaciones en el puesto de trabajo, etc. En definitiva, se trata de conseguir que se produzca un proceso de integración óptimo y con la garantía de que las barreras comunicativas queden neutralizadas y tanto la empresa como el trabajador estén satisfechos.

\section{Acceso al empleo público}

El empleo público es el generado por la actividad de la Administración en cualquiera de sus niveles (estatal, autonómico, local), tanto para la realización de funciones públicas (funcionarios) como para el desempeño de tareas o funciones que no revisten tal carácter (personal laboral). La búsqueda de empleo en cualquiera de las diferentes Administraciones Públicas presenta, en general, características propias. Por un lado, la obligada planificación del empleo público, que habilita un amplio periodo de tiempo entre el anuncio de la vacante y la selección del trabajador para cubrirla. Y, por otro, la exigencia de superar un proceso selectivo predeterminado y complejo antes de trabajar.

A continuación, se señalan los pasos a seguir ante ofertas de empleo público:
- Como campo acotado para la búsqueda, se debe identificar la/s oferta/s de empleo público que puedan contener empleos adecuados. Suelen convocarse con carácter anual, al ser una figura intimamente vinculada a la de los presupuestos del organismo o corporación, y aparecen publicadas en el boletín oficial correspondiente (nacional, regional 0 provincial).

- La oferta de empleo público contendrá, al menos, una referencia a las posibilidades de acceso de personas con minusvalía (reserva de plazas, adaptaciones, etc.). Sin embargo, raramente especificará cuántas plazas, de qué cuerpos o categorías laborales están reservadas.

- Publicada la oferta de empleo público, se ha de concretar el cuerpo o escala de funcionarios o categoría profesional a la que la persona con discapacidad auditiva va a intentar acceder, en función de su formación académica, su experiencia, sus preferencias y sus capacidades.

- Desde la publicación de la oferta de empleo público hasta la celebración de las pruebas suelen transcurrir unos meses, que pueden resultar idóneos para recibir formación teórica y/o práctica específica. No obstante, en función de los requerimientos del empleo ofertado, el plazo citado puede resultar insuficiente y puede ser necesario recurrir a acciones ante las diferentes administraciones para conocer las futuras convocatorias con la anticipación precisa para abordar acciones formativas eficaces. En ese sentido, cabe recordar que para algunas oposiciones es habitual prepararse durante más de un año (en general, las que exigen titulación superior). 
- Tras la publicación de cada oferta de empleo, se inicia la convocatoria de cada uno de los diversos procesos selectivos en ella previstos. Esta convocatoria conlleva la publicación de las bases, que especifican los requisitos exigidos por el cuerpo 0 escala de funcionarios o categoría profesional y describen y regulan las pruebas selectivas.

- Junto a la convocatoria se publica el impreso de solicitud de participación en las pruebas selectivas, que ha de contener espacios especificos para optar a una plaza reservada y para solicitar adaptaciones de tiempo y medios para la realización de los ejercicios, en el caso de que la convocatoria contenga reserva de plazas. Tal extremo, con precisión de las plazas reservadas, ha de constar en la propia convocatoria 0 en las bases de la misma, que también ha de indicar expresamente la forma de optar a tal reserva, así como la forma de solicitar adaptaciones para realizar las pruebas.

- Respecto a los requisitos exigidos, las bases suelen referirse a los requerimientos funcionales de los aspirantes en los siguientes términos: "No padecer limitación física, psiquica o sensorial que sea incompatible con el desarrollo de las correspondientes funciones". Aunque, a veces, se hace en términos menos integradores "no padecer enfermedad o defecto físico o psíquico que sea incompatible con el normal desarrollo de las correspondientes funciones". Son preferibles fórmulas neutras tales como "poseer capacidad funcional suficiente para desarrollar el trabajo ofrecido". Si bien, sólo excepcionalmente, aún pueden encontrarse cuadros de exclusiones médicas específicas para un cuerpo determinado (por ejemplo, cuerpos de instituciones penitenciarias) y, ocasionalmente, se encuentran bases que simplemente describen las prestaciones exigidas (determinada altura 0 determinada velocidad a la carrera).

- Desde la publicación de la convocatoria hay un plazo (normalmente, de entre 10 Y 20 dias) para solicitar la admisión a las pruebas selectivas, mediante la cumplimentación del impreso de solicitud:

- No suelen exigirse documentos en esta fase. Basta hacer constar en el impreso que se reúnen los requisitos exigidos, excepto para la exención de tasas de derecho de examen de la que pueden beneficiarse las personas con discapacidad igualo superior al 33\%, para lo que se exige certificación acreditativa de dicha condición.

- En el propio impreso ha de indicarse si se opta a plaza reservada, cuando las haya, y solicitar las adaptaciones correspondientes.

- En la celebración de las pruebas, el tribunal debe facilitar las adaptaciones que se hayan admitido. Si se le suscitan al Tribunal dudas sobre la capacidad funcional del aspirante en relación a la requerida por el empleo o sobre la idoneidad de la adaptación solicitada, puede recabar informes y/o colaboración de los organismos competentes.

- Superadas las pruebas, y en su caso, el curso selectivo, se publica la relación de aprobados, que también debe ser distinta para los aspirantes a plazas reservadas, siendo 
éste el momento de acreditar que se reúnen los requisitos exigidos en las bases.

En este sentido y desde nuestro SIOS, se ha informado de diferentes procesos selectivos a usuarios que en su perfil habían mostrado interés y aptitudes en este sentido e incluso a los que nosotros considerábamos capacitados para acceder a este tipo de plazas aunque ellos no lo hubieran solicitado expresamente.

\section{OTROS AGENTES IMPLICADOS EN LA INSERCIÓN LABORAL DE PERSONAS CON DISCAPACIDAD}

Además de los agentes mencionados en los capítulos anteriores, se pueden nombrar muchos otros que también están implicados en el proceso de inserción laboral, algunos de los cuales los vamos a detallar a continuación.

Son, Organizaciones Empresariales, las asociaciones creadas con la finalidad de defender y promover los derechos de los empresarios. En el terreno de la inserción laboral de personas con discapacidad, las asociaciones empresariales pueden contribuir, mediante el diálogo con la Administración, el asociacionismo y los sindicatos, a una más efectiva aplicación de la normativa en las empresas. Así mismo, pueden influir positivamente entre sus asociados y patrocinados a los efectos de aumentar su receptividad al empleo de personas con discapacidad. En este sentido, nosotros colaboramos, a través de diversos foros, con Asociaciones de Empresarios, o incluso con Asociaciones de Profesionales que trabajan directamente en el asesoramiento a empresas.

Por otra parte y como es sabido, los Sindicatos son asociaciones de trabaja- dores que tienen por objeto la defensa y promoción de sus derechos. En esta linea, los sindicatos pueden incidir en la inserción de las personas con discapacidad, al menos, desde tres puntos de vista:

- Con su actividad en relación con propuestas de nueva regulación normativa o de mejora de la misma.

- Mediante políticas de concienciación de los trabajadores, especialmente de sus afiliados.

- A través de la actuación de los representantes de los trabajadores en la empresa.

Por nuestra parte, tuvimos estrecha colaboración por parte de grandes sindicatos como UGT y especialmente $\mathrm{CCOO}$, quienes difundieron nuestro Proyecto entre sus afiliados y colaboraron a través de sus propios Servicios de Empleo. También y en casos puntuales, con sindicatos específicos (por ejemplo SATSE) cuando nuestros usuarios lo requerían.

Las Cámaras de Comercio son entidades corporativistas de las patronales creadas para fomentar el desarrollo de la economía y proteger los intereses de los empresarios. Elaboran memorias y estadísticas sobre la situación económica y tienen una red asociativa de comerciantes y empresarios con los que se puede contactar para informar sobre el Servicio de Empleo (objetivos, acciones, etc.), sobre las capacidades y potencialidades de las personas con discapacidad auditiva, sobre la normativa vigente en materia de contratación de estas personas, subvenciones, bonificaciones, etc. En este sentido, nosotros mantuvimos un estrecho contacto con las Cámaras de Comercio del Principado de Asturias, especialmente por su receptividad, con la Cámara de Comercio de Oviedo, que a 
través de su revista difundió nuestro Proyecto.

\section{Los Servicios Públicos de Empleo} son oficinas públicas de atención a los demandantes y ofertantes de empleo que dependen, en su mayoría, de la Consejería de Trabajo o Empleo de cada Comunidad Autónoma. La inscripción como demandante de empleo conlleva una serie de requisitos y documentos a aportar para poder acceder a los siguientes servicios:

- Registro como demandante de empleo.

- Información y acceso a las ofertas de empleo vigentes.

- Información sobre medidas de fomento de empleo (autoempleo, sociedades laborales, modalidades de contratación).

- Información sobre formación profesional y ocupacional (Plan Nacional de Formación e Inserción Laboral FIP, Talleres de Empleo, Escuelas Taller y Casas de Oficio).

- Información de prestaciones y subsidios por desempleo.

- Información y tramitación, en su caso, de la Renta Activa de Inserción.

- Orientación profesional.

- Oferta de empleo procedente de otros países de la Unión Europea (Red EURES).

En los Servicios Públicos de Empleo, captamos algunas de las ofertas de empleo de empresas con las que luego contactamos o hicimos llegar la oferta a nuestros usuarios directamente. Tam- bién algunos de nuestros usuarios participaron en alguna Escuela Taller a través de un contrato en esta modalidad y compartimos con ellos asesoramiento entorno a la personas con deficiencia auditiva cuando así lo requerían.

Los Institutos de Secundaria son otros de los agentes implicados en la inserción laboral y, por ello, el trabajo conjunto con el Servicio de Empleo debe tener como objetivo mejorar el nivel formativo de los jóvenes con discapacidad auditiva, requiriendo las adaptaciones y recursos de apoyo necesarios para el mantenimiento y éxito de estos alumnos en sus estudios.

Se realizarán acciones diversas como:

- Informar al alumnado con discapacidad auditiva, a sus familias, orientadores y profesores sobre los objetivos y acciones del Servicio de Empleo, facilitando el proceso de transición de la escuela al trabajo.

- Intercambiar información sobre los procesos de formación y empleo (retroalimentación).

- Concienciar a los centros educativos para que trabajen de forma transversal las habilidades sociales y laborales de los alumnos con discapacidad auditiva.

- Informar sobre los recursos existentes para la eliminación de las barreras de comunicación en las situaciones de enseñanza/aprendizaje.

- Trabajar conjuntamente con los departamentos de orientación para que los alumnos elijan opciones formativas adecuadas a sus preferencias, posibilidades y potencialidades así como a la realidad del mercado laboral actual. 
- Ofrecer el apoyo y seguimiento a las prácticas formativas en empresa de los ciclos formativos y de garantía social, colaborando con el tutor del centro y la empresa.

- Contactar con las AMPAS de los institutos (Asociaciones de Madres y Padres de los Alumnos) para ofrecerles información general sobre el Servicio de Empleo y sobre la discapacidad auditiva.

Nuestro SIOS, en su origen, estuvo estrechamente ligado a este campo, pues estaba financiado por el MEC. Por tanto, contactamos con todos los IES que algún momento cercano habian tenido o tenían alumnos con alguna deficiencia auditiva. Con ellos compartimos orientación y acompañamiento o gestión directa de prácticas de diferentes Ciclos Formativos y conseguimos que diferentes empresas diesen continuidad a estas prácticas educativas en contratos para nuestros usuarios.

Finalmente, no debemos olvidar la importancia de coordinarse con otros Proyectos de Empleo dedicados a la inserción de personas con discapacidad. Primero porque todos trabajamos con un mismo objetivo, segundo porque nuestros usuarios pueden participar de otros Proyectos que les ayuden en su búsqueda de empleo y tercero porque nuestra prospección laboral puede derivar en ofertas que no se adapten al perfil de nuestros usuarios pero sí al de otras personas con discapacidad en búsqueda de empleo.

\section{IMPORTANCIA DEL PAPEL DE LA FAMILIA EN EL PROCESO DE INTEGRACION LABORAL DE LA PERSONA SORDA}

El objetivo final del Servicio de Empleo es mejorar las condiciones de ocupabilidad de las personas con discapacidad auditiva para acceder al mercado de trabajo ordinario y, para ello, es necesaria la participación, no sólo de éstas, sino de todos los agentes implicados en el proceso: empleadores, centros formativos y, principalmente, del entorno más inmediato, la familia.

Por tanto, la labor de información y apoyo desarrollada con las familias es uno de los condicionantes decisivos para la más adecuada inserción laboral del joven sordo, centrándose esta labor en informar y orientar a los padres, concienciándoles de la importancia del empleo para sus hijos e implicando a las familias en el proceso de búsqueda activa de empleo e inserción laboral. Fundamentalmente, se realizan acciones que provoquen un cambio de actitud que fomente la autonomía personal del joven sordo con el apoyo familiar.

La familia, en general y en particular el caso de la discapacidad auditiva, se erige como una variable decisiva a la hora de que el joven cuente con un grado de autonomía personal y de recursos suficientes, así como para que disponga de los hábitos laborales y habilidades sociales que requiere el acceso a un puesto de trabajo.

Así, un entorno familiar sobreprotector, en el que la discapacidad del hijo desde su infancia sea considerada como invalidante para muchas actividades es, quizá, el elemento que más determina la actitud de la persona sorda frente a su propia vida. En estos casos, la familia aparece como una instancia que plantea que la persona con discapacidad auditiva necesita siempre estar acompañado por algún oyente, que prácticamente en cualquier actividad de su vida diaria el sordo requiere de ayuda para poder desenvolverse, que necesita ayuda para las entrevistas, etc. Esta actitud familiar limita la 
autonomía de la persona con este tipo de discapacidad, le genera una pérdida de confianza en sí misma y le hace más dependiente. Estas limitaciones se proyectan, sin duda alguna, al mundo del trabajo, a los propios procesos de inserción laboral en los que, en bastantes casos, el joven en busca de empleo mantiene una actitud pasiva y demandante.

Por el contrario, un entorno familiar involucrado y motivador, en el que el joven sordo recibe el apoyo necesario para superar, en la medida de lo posible, su discapacidad y en el que se le motive para conseguir una vida lo más independiente posible, dará lugar a unos esquemas mentales de funcionamiento y a unas actitudes más facilitadoras de la inserción laboral.

Como vemos, la implicación de la familia será lo que determine, en muy buena parte, la actitud del joven con discapacidad auditiva hacia la búsqueda de empleo.

La metodología a aplicar para el trabajo con las familias, ya sea de jóvenes o de adultos, ha de centrarse en el diálogo y la comunicación positiva. A continuación, se describen los objetivos a tener en cuenta en la intervención con la familia:

- Informar sobre las prestaciones del Servicio de Empleo, de manera que se promueva la credibilidad del mismo, tanto personal e institucional como de procedimiento.

- Promover la confianza y credibilidad hacia una futura vida laboral de sus hijos.

- Aumentar el compromiso de favorecer la incorporación de sus hijos al mercado laboral.

- Aprovechar las oportunidades para los refuerzos positivos y el reconocimiento de determinadas acciones de sus hijos.

- Concienciar a las partes de la importancia del diálogo, la cooperación y el compromiso.

- Corresponsabilizar a ambas partes en el proceso, en la medida en que a cada una le corresponda.

- Evaluar continuamente el proceso, detectando los posibles cambios de actitud que puedan generarse.

Ni que decir tiene que un Proyecto promovido por un Asociación de Padres de Personas con Deficiencia Auditiva tenia muy presente este aspecto que no se observa en otros Proyectos de Empleo y que sin embargo desde nuestro experiencia podemos afirmar que puede condicionar una inserción laboral exitosa. Por tanto, conocedores de este aspecto, trabajamos con cada una de las familias de nuestros 114 usuarios en "Bolsa de Empleo", en todos los aspectos antes referidos y adaptados a cada caso concreto. Fundamentalmente y como anteriormente hemos referido trabajábamos con usuarios muy jóvenes que hacian su transición de la escuela al trabajo y este es un momento crítico para ellos y su familia. Pero también, otros de nuestros usuarios eran perceptores de pensiones y estaban en formación ocupacional por lo que en esta cultura tan española "de la pensión y el subsidio" temían perder su seguridad en aras de un contrato en algunos casos inestable y con ingresos básicos, aunque siempre superiores a lo que en su momento percibian.

\section{INDICADORES OBJETIVAMENTE VERIFICABLES}

Una vez expuesto nuestra metodología 
de trabajo expondremos los resultados cuantificables en contratos conseguidos con el Proyecto, aunque sin olvidar que hay mucho otro trabajo muy difícil de cuantificar y otros resultados que se dilatarán en el tiempo aunque serán función directa de nuestro trabajo.

El SIOS de APADA-ASTURIAS inició su andadura bajo supervisión de FIAPAS (Federación Española de Padres y Amigos de los Sordos). FIAPAS como Federación coordinó varios Proyectos de empleo en diferentes Asociaciones de Padres de deficientes auditivos y obtuvo en conjunto los siguientes resultados (ver tabla 1): pues el $72 \%$ de los contratos son de seis o más meses de duración y suponen una cierta estabilidad.

Por nuestra parte, el SIOS de APADAASTURIAS inició su andadura en PILII y mi colaboración personal en dicho Proyecto finalizó en marzo de 2002, pero también obtuvimos unos resultados que paso a exponerles en la tabla 2.

De los datos cuantitativos que se refieren a nuestro Proyecto nos gustaría resaltar:

1. El aumento significativo de los con-

Tabla 1. Número de contratos conseguidos en FIAPAS

\begin{tabular}{|l|l|c|c|c|}
\hline \multicolumn{1}{|c|}{ Proyecto } & \multicolumn{1}{|c|}{ Periodo } & Total contratos & Total ordinaria & $\begin{array}{c}\text { Ordinaria }+ \text { de } \\
6 \text { meses }\end{array}$ \\
\hline PIL-I & $1996 / 97$ & 114 & $76(67 \%)$ & $54(71 \%)$ \\
\hline PIL-II & $1998 / 99$ & 340 & $302(89 \%)$ & $219(73 \%)$ \\
\hline PIL-III & 2000 & 214 & $176(82 \%)$ & $155(88 \%)$ \\
\hline DINAMI & $1998 / 00$ & 330 & $272(82 \%)$ & $180(66 \%)$ \\
\hline FIAPAS & 2001 & 239 & $204(83 \%)$ & $130(64 \%)$ \\
\hline TOTALES & & 1237 & $1030(83 \%)$ & $738(72 \%)$ \\
\hline
\end{tabular}

Como se puede observar, la progresión en número de contratos y en porcentajes de contratos en empresa ordinaria, en general, y de contratos en empresa ordinaria de más de seis meses es notable. Y refleja, en primer lugar, el éxito del Proyecto a nivel nacional y la pertinencia de mantener estos Proyectos a largo plazo pues muestran una progresión en los resultados apreciable e interesante de mantener para las personas con deficiencia auditiva. Concretamente, más de 8 de cada 10 empleos corresponden a actividades laborales ordinarias y sólo el $17 \%$ a actividades protegidas. Además, se trata de empleo de calidad, tratos en empresa ordinaria de larga duración (seis meses o más incluidos contratos indefinidos) del PIL II al PIL III. En cuanto al último periodo (referido como propiamente SIOS), el número de contratos es marcadamente inferior al de otros periodos pero este Proyecto es de duración mucho menor, siete meses frente a dos años que incluían el periodo vacacional, momento durante el cual la oferta de trabajo aumentaba significativamente.

2. Por otra parte, los usuarios que permanecian en nuestra bolsa de 
Tabla 2. Número de contratos conseguidos en ADAPA

\begin{tabular}{|l|l|c|c|c|}
\hline \multicolumn{1}{|c|}{ Proyecto } & \multicolumn{1}{|c|}{ Periodo } & Total contratos & Total ordinaria & $\begin{array}{c}\text { Ordinaria + de } \\
6 \text { meses }\end{array}$ \\
\hline PIL-II & $1998 / 99$ & 45 & $30(67 \%)$ & $14(73 \%)$ \\
\hline PIL-III & $2000 / 01$ & 53 & $38(72 \%)$ & $35(92 \%)$ \\
\hline SIOS & $\begin{array}{l}\text { Agosto 2001- } \\
\text { Marzo 2002 }\end{array}$ & 5 & $5(100 \%)$ & $2(40 \%)$ \\
\hline TOTALES & & 103 & $73(71 \%)$ & $51(70 \%)$ \\
\hline
\end{tabular}

empleo e incluso los que se habian incorporado estaban menos cualificados que los que ya habían encontrado empleo y mostraban minusvalias mucho más limitantes para el desenvolvimiento laboral y por tanto su inserción era tanto más laboriosa. Sin embargo, todas las contrataciones de este periodo han sido en el entorno ordinario dejando de lado el ámbito protegido.

3. Además, mantuvimos el seguimiento y consolidación de los puestos de larga duración conseguidos a través de PIL II y PIL III, junto con los conseguidos durante el SIOS subvencionado por el Principado. Pues bien, la gran mayoría de ellos se han mantenido e incluso transformado en indefinidos cuando ha existido voluntad por parte del joven con deficiencia auditiva.
Finalmente, nos gustaria resaltar la puesta en marcha de una empresa bajo nuestra supervisión y asesoramiento por parte de uno de nuestros usuarios, que posiblemente derive en contrataciones a otras personas con minusvalía, aspecto que nos gratifica especialmente.

Todos los datos anteriormente expuestos reflejan a nuestro modo de ver:

1. La capacidad de las personas con deficiencia auditiva para competir con éxito en el mercado de trabajo ordinario, en virtud de sus propios méritos y con un poco de asesoramiento y acompañamiento.

2. La necesidad de estabilidad de estos proyectos dada la progresión de los resultados obtenidos.

\section{BIBLIOGRAFÍA}

Constitución Española de 1978.

FIAPAS (2004). "Guía de Buenas Prácticas para los Servicios de Empleo" de la Red de Inserción Laboral de FIAPAS. Madrid, FIAPAS.

Ley 13/1982, de 7 de Abril, de Integración Social de los Minusválidos (LISMI).
Ministerio de Educación y Cultura. Orden de 23 de noviembre de 1998, BOE 26 de enero de 1999.

Ministerio de Educación y Cultura. Orden 28 de abril de 1999, BOE 27 de mayo de 1999.

Plan de Acción para el Empleo del Reino de España 1998. 